\title{
MobileCoDaC - a transportable Control, Data Acquisition and Communication Infrastructure for Wendelstein 7-X
}

\author{
Christine Hennig ${ }^{,},{ }_{1}$ Torsten Bluhm ${ }^{l}$, Georg Kühner ${ }^{1}$, Heike Laqua ${ }^{l}$, Marc Lewerentz ${ }^{1}$, Ina Müller ${ }^{1}$, \\ Steffen Pingel ${ }^{I}$, Heike Riemann ${ }^{l}$, Jörg Schacht ${ }^{l}$, Anett Spring ${ }^{l}$, Andreas Werner ${ }^{l}$, Andreas Wölk ${ }^{l}$ \\ ${ }^{\text {I} M a x-P l a n c k-I n s t i t u t ~ f u ̈ r ~ P l a s m a p h y s i k, ~ G r e i f s w a l d, ~ G e r m a n y ~}$ \\ *corresponding author Christine.Hennig@ipp.mpg.de
}

MobileCoDaC is a test bed allowing in situ testing and commissioning the control and data acquisition of components to be operated at Wendelstein 7-X. It is a minimized replica of the functionality of the complete $\mathrm{W} 7-\mathrm{X} \mathrm{CoDaC}$ infrastructure and can be operated independently.

MobileCoDaC contains a set of W7-X CoDaC servers, network infrastructure, and accessories for remote access. All hardware is mounted in a single transportable rack system. Moreover, it provides the software infrastructure and user applications for experiment preparation, experiment operation, trouble shooting and experiment data access.

MobileCoDaC has been operated successfully for test and commissioning of the control and data acquisition of the HEXOS (high efficiency extreme ultraviolet overview spectrometer) diagnostic at Forschungszentrum Jülich.

Keywords: CoDaC, Control, Data Acquisition, in situ, Test, Testbed, Infrastructure 


\section{Introduction}

\subsection{The Wendelstein 7-X fusion experiment}

The Max-Planck-Institut für Plasmaphysik (IPP) investigates the principles underlying a power plant, which - like the sun - will produce energy from the fusion of light atomic nuclei. Wendelstein 7-X (W7-X) is a stellarator with modular superconducting coils which enable steady state plasma and is the only one of its kind in the world. For scientific experimentation of $\mathrm{W} 7-\mathrm{X}$ a tailored control, data acquisition and communication $(\mathrm{CoDaC})$ system has been developed.

\section{$1.2 W 7-X \mathrm{CoDaC}$}

W7-X CoDaC develops and provides all necessary hard- and software for machine and experiment control, data acquisition and database services as well as the communication and IT infrastructure. It supports scientific experimentation as well as technical operation of W7-X. Its unique characteristic is the support of continuous (up to 30 minutes) as well as short-pulse (some seconds) experiment operation.

W7-X CoDaC consists of a distributed component system where all subsystems (diagnostics, plasma heating etc.) act as single components interacting with each other. Operation is performed by running preconfigured segments. All data is logged with experiment wide synchronized timestamps. Data acquisition with high resolution for scientific data during experiments as well as long-term recording of operational data up to 24 hour/7days is supported. Online monitoring for trending, which is indispensable for continuous operation, is provided. Service oriented online and offline data analysis is integrated as well. This provides access to the results of plasma theory and plasma modeling and, furthermore, access to the highly sophisticated machine geometry data. Uniform data retrieval of measured and analyzed data, allowing time and structure information as selection criteria, has been implemented. A recent description of the challenges mastered can be found in [1].

\section{3. (Remote) commissioning of a component using MobileCoDaC}

Commissioning of W7-X components is done in several collaboration projects within the scientific fusion community and much of this work is done at remote sites. Therefore, the necessity of a transportable and minimalistic W7-X CoDaC test bed (MobileCoDaC) has been emerged. However, from the very first beginning, W7-X CoDaC development had a fully integrated approach with high cohesion to the local IT infrastructure.

The MobileCoDaC challenge can be characterized in a single question: "How to ship a replica of a complex software and hardware environment together with a complete IT infrastructure to a remote site, all fitting in a transportable, mobile rack?"

To achieve this, a review of all W7-X CoDaC software and hardware components as well as the related IT infrastructure has been conducted with respect to isolating functionality and reducing physical dimension. Moreover, MobileCoDaC has been reduced as much as possible in functionality so that only the absolutely indispensable parts for commissioning have been replicated.

A typical component under test has a dedicated control and data acquisition system consisting of a PLC for slow control and two control stations of type FCS for fast control and of type $\mathrm{CoDa}$ for data acquisition. Correct operation of these items and their interaction needs to be verified. Therefore, they have to be connected to MobileCoDaC and operated accordingly.

The first component under test that has been tested and commissioned in combination with MobileCoDaC is the control and data acquisition of the HEXOS diagnostic [2]. This is also referred to "component under test" within this paper.

\section{Mobile CoDaC description}

This chapter describes MobileCoDaC and focuses on the similarities and differences to W7-X CoDaC.

\subsection{MobileCoDaC requirements}

In order to define the $\mathrm{W} 7-\mathrm{X} \mathrm{CoDaC}$ features absolutely necessary for in situ tests of a component under test, a precise requirements 
analysis has been accomplished. Table 1 shows the results of the requirements elicitation regarding the nonfunctional requirements.

\begin{tabular}{|l|l|l|}
\hline $\begin{array}{l}\text { Non Functional } \\
\text { Requirements }\end{array}$ & $\begin{array}{l}\text { Mobile } \\
\text { CoDaC }\end{array}$ & $\begin{array}{l}\text { W7-X } \\
\text { CoDaC }\end{array}$ \\
\hline Installation space & minimal & - \\
\hline $\begin{array}{l}\text { Transportable } \\
\text { Rack System }\end{array}$ & yes & - \\
\hline $\begin{array}{l}\text { Reusability for other } \\
\text { Commissioning Tests }\end{array}$ & yes & yes \\
\hline $\begin{array}{l}\text { IP Multicast for } \\
\text { Internal Communication }\end{array}$ & yes & yes \\
\hline $\begin{array}{l}\text { Logging for } \\
\text { Trouble Shooting }\end{array}$ & yes & yes \\
\hline Standalone Operation & yes & no \\
\hline $\begin{array}{l}\text { Integration into external } \\
\text { IT Infrastructure }\end{array}$ & yes & no \\
\hline $\begin{array}{l}\text { Remote Access } \\
\text { from IPP }\end{array}$ & yes & - \\
\hline Local Access in situ & yes & - \\
\hline
\end{tabular}

Table 1 Non functional requirements for MobileCoDaC compared to W7X CoDaC.

A system and software architecture for MobileCoDaC following the arc42 template [3] has been defined accordingly. The use of the $\operatorname{arc} 42$ template was extremely helpful in structuring the review process.

\subsection{MobileCoDaC concept}

One key concept was to use virtual servers for all services in order to reduce physical size. This was verified successfully in an evaluation phase with respect to potential bandwidth issues.

The use of the W7-X CoDaC system requires a lot of expert knowledge. In order to provide online help for the user, a possibility for remote access, remote troubleshooting and remote maintenance of MobileCoDaC and the component under test is indispensable and has been added accordingly.

Test plans conforming to the IEEE 829 Standard for Software and System Test Documentation [4] have been defined and documented and tests have been conducted accordingly, thus guaranteeing the high quality of the deliverable, see also section 3 .
MobileCoDaC hardware is designed to fit in a rollable 19" rack system. Table 2 lists the hardware items mounted in the MobileCoDaC installation (the subset below Part 1) and the items of the component under test (the subset below Part 2) that are connected to MobileCoDaC but not part of MobileCoDaC itself.

\begin{tabular}{|l|l|l|l|}
\hline Item & Purpose & LAN & TTE \\
\hline $\begin{array}{l}\text { Part 1: } \\
\text { Mobile CoDaC }\end{array}$ \\
\hline PC1 & virtual servers & $\mathrm{x}$ & - \\
\hline PC2 & time central & $\mathrm{x}$ & $\mathrm{X}$ \\
\hline & $\begin{array}{l}\text { user PC and } \\
\text { troubleshooting }\end{array}$ & $\mathrm{x}$ & - \\
\hline PC3 & switch & $\mathrm{x}$ & - \\
\hline SW & router & $\mathrm{x}$ & - \\
\hline ROUT & TTE switch & - & $\mathrm{X}$ \\
\hline SW-TTE & KVM over IP & $\mathrm{x}$ & - \\
\hline Accessory1 & power over IP & $\mathrm{x}$ & - \\
\hline Accessory2 & Power| & \multicolumn{2}{|l}{} \\
\hline Part 2: \\
Component under test & \multicolumn{5}{|l}{} \\
\hline PLC & slow control & $\mathrm{x}$ & - \\
\hline FCS & fast control & $\mathrm{x}$ & $\mathrm{X}$ \\
\hline CoDa & data acquisition & $\mathrm{x}$ & $\mathrm{X}$ \\
\hline
\end{tabular}

Table 2 hardware deliverable items with respect to the network connections of MobileCoDaC and the component under test

\subsection{MobileCoDaC PCs}

The number of server PCs to deliver could be reduced to $3 \mathrm{PCs}$ in total, see table 2:

(PC1) All central IT services have been virtualized on a single host $\mathrm{PC}$ using the Vmware ESX technology [5].

(PC2) A dedicated PC acts as W7-X time server providing the central synchronized high resolution time. This PC cannot be virtualized because it needs a dedicated hardware TTE central PCI card [6] and a real time operating system.

(PC3) Another PC has been added for user access and troubleshooting. It is separated from PC1, thus allowing trouble shooting also PC1.

\subsection{MobileCoDaC networks}

There are two networks installed in MobileCoDaC, see also table 2. In contrast to $\mathrm{W} 7-\mathrm{X} \mathrm{CoDaC}$ there is no additional dedicated real time network supplied. 


\subsubsection{Trigger Time Event network (TTE)}

The TTE network [6] has been installed identical to W7X-TTE in the MobileCoDaC rack. It consists of a unidirectional dedicated proprietary TTE optical fiber network and is connected by means of the TTE switch (SWTTE) to the time server (PC2) and the component under test.

Clients can get the precise synchronized time via the dedicated TTE network. A Client PC needs a TTE or TDC device for that. Typical clients are the control stations of the component under test.

Additionally, lower accuracy synchronized time is supplied using NTP over the LAN. Typical NTP clients are PLCs of the component under test as well as user applications.

\subsubsection{Local Area Network (LAN)}

To fulfill one of the key infrastructure requirements (table 1) a dedicated LAN is installed in the MobileCoDaC rack, see table 2. The LAN is provided and configured by the switch (SW) and allows routing the required UDP/IP Multicast traffic that is needed to communicate with the component under test.

The switch chosen is an Ethernet layer2 switch with sixteen 1Gbit/s and a single 10Gbit/s slots. It guarantees the required bandwidth for HEXOS data acquisition.

All MobileCoDaC PCs and the component under test are connected to this MobileCoDaC LAN. It can be operated completely independent of the network at the remote site.

A supplemented network router (ROUT) allows connection to the remote site and the IPP home site.

\subsection{MobileCoDaC virtual servers}

All virtual servers are running at a single host machine PC1 (table 2). A list of the required IT infrastructure is shown in table 3 . A dedicated Windows Domain Server has been added to supporting the isolated operation of MobileCoDaC. All other services are available at W7-X CoDaC and needed to be replicated for and adapted to the MobileCoDaC domain service.

\begin{tabular}{|l|l|l|}
\hline Server Infrastructure & MobileCoDaC & $\begin{array}{l}\text { W7-X } \\
\text { CoDaC }\end{array}$ \\
\hline Windows Domain Server & added & - \\
\hline Configuration Database & adapted & available \\
\hline Archive Database & adapted & available \\
\hline $\begin{array}{l}\text { Data Access Web } \\
\text { Service }\end{array}$ & adapted & available \\
\hline Messages Server & adapted & available \\
\hline Software Deploy Server & adapted & available \\
\hline $\begin{array}{l}\text { Runtime Environment } \\
\text { for Control Stations }\end{array}$ & adapted & available \\
\hline
\end{tabular}

Table 3 List of infrastructure servers

\subsection{The user application software}

MobileCoDaC provides a reduced set of user application software for commissioning and operation of the component under test. Operation with MobileCoDaC is nearly identical to operation at the W7-X machine and within the W7-X environment. Table 4 lists the application software delivered with MobileCoDaC categorized by use case as well as the software that has been omitted.

\begin{tabular}{|c|c|c|}
\hline Software & MobileCoDaC & $\begin{array}{l}\text { W7-X } \\
\text { CoDaC }\end{array}$ \\
\hline \multicolumn{3}{|c|}{ experiment preparation } \\
\hline Confix & - & available \\
\hline $\begin{array}{l}\text { Configuration } \\
\text { Editor }\end{array}$ & $\begin{array}{l}\text { adapted, } \\
\text { added examples }\end{array}$ & available \\
\hline Xedit & $\begin{array}{l}\text { adapted, } \\
\text { added examples }\end{array}$ & available \\
\hline \multicolumn{3}{|c|}{ experiment operation } \\
\hline $\begin{array}{l}\text { Monitor } \\
\text { System }\end{array}$ & - & available \\
\hline Xcontrol & $\begin{array}{l}\text { adapted, } \\
\text { added examples }\end{array}$ & available \\
\hline Logbook & $\begin{array}{l}\text { adapted, } \\
\text { added examples }\end{array}$ & available \\
\hline \multicolumn{3}{|c|}{ trouble shooting } \\
\hline Xnote & no adaptations & available \\
\hline \multicolumn{3}{|l|}{ data access } \\
\hline DataBrowser & no adaptations & available \\
\hline $\begin{array}{l}\text { Data Access } \\
\text { Framework }\end{array}$ & $\begin{array}{l}\text { adapted, } \\
\text { added examples }\end{array}$ & available \\
\hline $\begin{array}{l}\text { Analysis } \\
\text { Framework }\end{array}$ & - & $\begin{array}{l}\text { partial } \\
\text { available }\end{array}$ \\
\hline
\end{tabular}

Table 4 MobileCoDaC and W7-X CoDaC user application software 
The following adaptations of the software for MobileCoDaC have been necessary:

(1) Sample configurations and segments for the control stations have been defined and are provided to be used for the component under test.

(2) Software has been adapted to interoperate with the MobileCoDaC infrastructure instead of the W7-X CoDaC infrastructure

This improved the applications overall quality by adding customizability and increasing portability.

\section{7 (Remote) access possibilities for maintenance of MobileCoDaC}

One of the main challenges of MobileCoDaC is to provide an independent isolated LAN configuration allowing both isolated and remote operation.

To provide remote access via internet, a router has been added to the deliverable. The router can be configured freely according to the needs and policy of the cooperating institution.

All PCs and devices from MobileCoDaC can be operated remote via TCP/IP, including keyboard and power supply.

For local in situ access a user PC with KVM (keyboard, video, and mouse) console (Accessory1, see table 2) is provided to operate MobileCoDaC and the component under test. All other servers and all software are reachable from the user PC inside MobileCoDaC. The $\mathrm{KVM}$ can be controlled remotely.

In worst case if a reboot of a PC is necessary the power can be switched off and switched on remotely (Accessory2, see table 2).

All remote access features have been added solely for MobileCoDaC use.

\section{Test Plan for MobileCoDaC Commissioning}

Before shipping MobileCoDaC, it had to be ensured that it provides all W7-X CoDaC features required by the component under test.

W7-X CoDaC had successfully been used with WEGA as test bed [7] for a couple of years. Tests, troubleshooting and successful operation all was evolving simultaneously as the WEGA project itself improved.

On the other hand, there was no dedicated procedure available that could simply be reused for testing MobileCoDaC. Thus, appropriate tests needed to be defined, scheduled and executed.

\subsection{Standalone Tests}

When testing MobileCoDaC standalone it is not connected to any external network. However, it must be connected to the component under test. The MobileCoDaC networks are patched as marked in table 2 .

The standalone test suite comprises the following tests:

(1) MobileCoDaC infrastructure (login procedures etc.)

(2) Experiment preparation (find and read example configuration)

(3) Experiment operation (load and execute example configuration and segments)

(4) Data access (read archived operation execution data and measured data of the component under test)

(5) Data analysis (use sample programs provided to read and analyze archived data from the component under test)

(6) Remote trouble shooting

All standalone tests have been carried out successfully, both at the local site before shipping and at the remote site after shipping. Thus, the correct operation of MobileCoDaC in connection with control and data acquisition of a component under test is guaranteed.

\subsection{Remote Access Tests}

In order to testing the remote MobileCoDaC access it must be connected to an external network and accessed from outside this network.

The remote test suite is summarized in table 5 .

\begin{tabular}{|l|l|l|}
\hline Test no & Protocol & verification of \\
\hline (R1) & telnet & login to switch (SW) \\
\hline (R2) & sftp & $\begin{array}{l}\text { (PC3) authentication to } \\
\text { MobileCoDaC domain }\end{array}$ \\
\cline { 3 - 3 } & $\begin{array}{l}\text { (PC3) file transfer } \\
\text { remote site <-> IPP }\end{array}$ \\
\hline (R3) & rdp & $\begin{array}{l}\text { (PC3) access using } \\
\text { RemoteDesktop }\end{array}$ \\
\hline (R4) & http & $\begin{array}{l}\text { login to KVM } \\
\text { (Accessory1) }\end{array}$ \\
\hline (R5) & http & $\begin{array}{l}\text { login to PowerSwitch } \\
\text { (Accessory 2) }\end{array}$ \\
\hline (R6) & http & $\begin{array}{l}\text { login to ILO } \\
\text { of ESX server (PC1) }\end{array}$ \\
\hline
\end{tabular}


Table 5: Remote maintenance test plan

These tests have to be carried out at the remote site in order to guarantee the remote trouble shooting access.

All tests have been simulated at the local site.

\section{Related work}

ITER, the International Thermonuclear Experimental Reactor, has developed a MiniCODAC system. It implements the human machine interface according to ITER CODAC standardized interfaces. There is a high demand for such a MiniCODAC system resulting from the in kind procurement where seven domestic agencies are collaborating worldwide [8]. Central CODAC services are replaced by the Mini-CODAC system. MiniCODAC is both a system development and a runtime platform while providing a subset of CODAC central services [9]. Configuration developed in the Mini-CODAC environment can be used for factory acceptance test, site acceptance test and integrated operation.

This is in contrast to the integrated approach of W7-X CoDaC and MobileCoDaC.

While MiniCODAC is a pure Software deliverable, MiniCODAC++ does also provide necessary network infrastructure and is thus more comparable to MobileCoDaC.

Another pure software approach has long since been used in the JET machine, the Joint European Torus. JET CODAS defined a black box http protocol as user interface for any control and data acquisition program [10]. This serves as interface to the CODAS environment and does not require any hardware deliverable but offers less functionality than MobileCoDaC.

\section{Conclusions}

The challenges from the creation of an independent and transportable W7-X CoDaC environment for commissioning purposes have been mastered successfully in building, testing and shipping MobileCoDaC.

MobileCoDaC is an excellent tool to support remote collaboration with external partners. It allows testing the control and data acquisition of a component by providing a $\mathrm{W} 7-\mathrm{X} \mathrm{CoDaC}$ environment. Thus, correct interaction of the component under test with the central W7-X $\mathrm{CoDaC}$ services can be verified.

The following key improvements of the W7-X $\mathrm{CoDaC}$ have been reached when implementing MobileCoDaC:

(1) More stringent software deployment

(2) Dedicated commissioning procedures for single components under test

(3) Dedicated test plans for MobileCoDaC

(4) Improved hardware, software, admin and user documentation including FAQ

(5) Conformity marking for MobileCoDaC

(6) Remote access for trouble shooting MobileCoDaC

(7) Reproducibility

Moreover, MobileCoDaC allows intensive practicing of the $\mathrm{W} 7-\mathrm{X}$ CoDaC user application software and therefore is also an education toolset. Experience can be used for further component tests. The quality procedures ensure that MobileCoDaC passed intensive tests prior to shipping.

MobileCoDaC has successfully been used for test and commission the control and data acquisition of HEXOS at the remote site Forschungszentrum Jülich. It has been carried out together with the local customer and the W7-X CoDaC experts at the remote site.

Very special thanks to the colleagues in Jülich for their support and collaboration.

Smooth integration of the HEXOS control and data acquisition back into the W7-X CoDaC environment is anticipated with minimum effort.

\section{References}

[1] A. Werner, et al.

Cutting Edge Concepts for Control and Data

Acquisition for Wendelstein 7-X

http://dx.doi.org/10.1109/SOFE.2013.6635430

[2] J. Schacht, et al.

The implementation of the Wendelstein 7-X control a data acquisition concepts at VUV/XUV overview spectrometers HEXOS Fusion Engineering and Design http://dx.doi.org/10.1016/j.fusengdes.2012.05.015

[3] Starke, G. Hruschka, P. arc 42 template http://www.arc42.org/ 


\section{[4] IEEE 829}

http://en.wikipedia.org/wiki/IEEE_829

[5] VmWare

www.vmware.com

[6] J. Schacht, et al.

A trigger-time-event system for the W7-X

experiment

http://dx.doi.org/10.1016/S0920-3796(02)00035-2

[7] H. Laqua, et al.

Test of the steady state W7-X control and data acquisition system at the WEGA stellarator http://dx.doi.org/10.1016/j.fusengdes.2009.10.005

[8] S. Simrock, et al.

Integration of the ITER diagnostic plant systems with CODAC

http://dx.doi.org/10.1016/j.fusengdes.2011.01.101

[9] F. Di Maio, et al.

The CODAC software distribution for the ITER plant system http://accelconf.web.cern.ch/accelconf/icalepcs2011 /papers/mopks029.pdf

[10] Hogben, C. and Griph, S.:

Interfacing to JET Plant Equipment Using the HTTP Protocol; JDN/H(02)011; Issue $2: 14$ th August 2008

http://www.iop.org/Jet/fulltext/EFDR02004.PDF 